\title{
Biharmonic maps on kenmotsu manifolds
}

\author{
Abdelkader Zagane ${ }^{1}$ and Seddik Ouakkas ${ }^{2}$ \\ ${ }^{1}$ Department of mathematics - University of Mascara, Algeria \\ ${ }^{2}$ Department of mathematics - University of Saida, Algeria
}

Received: 27 January 2016, Accepted: 4 March 2016

Published online: 22 June 2016.

\begin{abstract}
In this paper we study biharmonic maps on Kenmotsu manifolds. An example for biharmonic map of a three-Kenmotsu
\end{abstract} manifold is constructed for illustration.

Keywords: Biharmonic map, Kenmotsu manifold, three-Kenmotsu manifold.

\section{Introduction}

\subsection{Kenmotsu manifolds.}

The notion of Kenmotsu manifolds is defined by K. Kenmotsu (see [15]). Let $\left(M^{2 n+1}, \phi, \xi, \eta, g\right)$ be an almost contact Riemannian manifold, where $\phi$ is an $(1,1)$ tensor field, $\eta$ is a 1-form and $g$ is the Riemannian metric. We have for any $X, Y \in \Gamma(T M)$ (see [7] and [21])

$$
\phi(\xi)=0, \eta(\phi(X))=0, \eta(\xi)=1
$$

$$
\phi^{2}(X)=-X+\eta(X) \xi
$$

$$
g(X, \xi)=\eta(X)
$$

and

$$
g(\phi(X), \phi(Y))=g(X, Y)-\eta(X) \eta(Y)
$$

If, moreover

$$
\left(\nabla_{X} \phi\right) Y=-\eta(Y) \phi(X)-g(X, \phi(Y)) \xi
$$

and

$$
\nabla_{X} \xi=X-\eta(X) \xi
$$

where $\nabla$ denotes the Riemannian connexion of $g$, then $\left(M^{2 n+1}, \phi, \xi, \eta, g\right)$ is called an almost Kenmotsu manifold. In kenmotsu manifolds, we have the following relations (see [15]) :

$$
\left(\nabla_{X} \eta\right) Y=g(\phi(X), \phi(Y))
$$




$$
\begin{gathered}
R(X, Y) \xi=\eta(X) Y-\eta(Y) X \\
R(\xi, X) Y=\eta(Y) X-g(X, Y) \xi \\
R(\xi, X) \xi=X-\eta(X) \xi
\end{gathered}
$$

and

$$
S(X, \xi)=-(n-1) \eta(X)
$$

where $R$ is the Riemannian curvature tensor and $S$ is the Ricci tensor. Kenmotsu manifolds have been studied by many authors, for example see [8], [14] and [20].

\subsection{Harmonic maps.}

Let $\phi:\left(M^{m}, g\right) \rightarrow\left(N^{n}, h\right)$ be a smooth map between riemannian manifolds. Then $\phi$ is said to be harmonic if it is a critical point of the energy functional :

$$
E(\phi)=\frac{1}{2} \int_{M}|d \phi|^{2} d v_{g}
$$

with respect to compactly supported variations. Equivalently, $\phi$ is harmonic if it satisfies the associated Euler-Lagrange equations :

$$
\tau(\phi)=\operatorname{Tr}_{g} \nabla d \phi=0
$$

$\tau(\phi)$ is called the tension field of $\phi$. One can refer to [1], [5], [9], [10], [11] and [12] for background on harmonic maps. Indeed, the Euler-Lagrange equation associated to the energy is the vanishing of the tension field $\tau(\phi)=\operatorname{Tr}_{g} \nabla d \phi$. In the context of harmonic maps, the stress-energy tensor was studied in details by Baird and Eells in [2]. Indeed, the EulerLagrange equation associated to the energy is the vanishing of the tension field $\tau(\phi)=\operatorname{Tr}_{g} \nabla d \phi$, and the stress-energy tensor for a map $\phi:\left(M^{m}, g\right) \longrightarrow\left(N^{n}, h\right)$ defined by

$$
S(\phi)=e(\phi) g-\phi^{*} h
$$

The relation between $S(\phi)$ and $\tau(\phi)$ is given by

$$
\operatorname{div} S(\phi)=-h(\tau(\phi), d \phi)
$$

\subsection{Biharmonic maps.}

The map $\phi$ is said to be biharmonic if it is a critical point of the bi-energy functional :

$$
E_{2}(\phi)=\frac{1}{2} \int_{M}|\tau(\phi)|^{2} d v_{g}
$$

Equivalently, $\phi$ is biharmonic if it satisfies the associated Euler-Lagrange equations :

$$
\tau_{2}(\phi)=-\operatorname{Tr}_{g}\left(\nabla^{\phi}\right)^{2} \tau(\phi)-\operatorname{Tr}_{g} R^{N}(\tau(\phi), d \phi) d \phi=0
$$


where $\nabla^{\phi}$ is the connection in the pull-back bundle $\phi^{-1}(T N)$ and, if $e_{i}$ is a local orthonormal frame field on $M$, then

$$
\operatorname{Tr}_{g}\left(\nabla^{\phi}\right)^{2} \tau(\phi)=\left(\nabla_{e_{i}}^{\phi} \nabla_{e_{i}}^{\phi}-\nabla_{\nabla_{e_{i}} e_{i}}^{\phi}\right) \tau(\phi)
$$

where we sum over repeated indices. We will call the operator $\tau_{2}(\phi)$, the bi-tension field of the map $\phi$. In analogy with harmonic maps, Jiang In [13] has constructed for a map $\phi$ the stress bi-energy tensor defined by

$$
S_{2}(\phi)=\left(\frac{-1}{2}|\tau(\phi)|^{2}+\operatorname{divh}(\tau(\phi), d \phi)\right) g-2 \operatorname{symh}(\nabla \tau(\phi), d \phi),
$$

where

$$
\operatorname{symh}(\nabla \tau(\phi), d \phi)(X, Y)=\frac{1}{2}\left\{h\left(\nabla_{X} \tau(\phi), d \phi(Y)\right)+h\left(\nabla_{Y} \tau(\phi), d \phi(X)\right)\right\},
$$

for any $X, Y \in \Gamma(T M)$. The stress bi-energy tensor of $\phi$ satisfies the following relationship

$$
\operatorname{div} S_{2}(\phi)=h\left(\tau_{2}(\phi), d \phi\right)
$$

Clearly any harmonic map is biharmonic, therefore it is interesting to construct non-harmonic biharmonic maps. In [4] the authors found new examples of biharmonic maps by conformally deforming the domain metric of harmonic ones. While in [6] the author analyzed the behavior of the biharmonic equation under the conformal change the domain metric, he obtained metrics $\widetilde{g}=e^{2 \gamma}$ such that the idendity map $I d:(M, g) \longrightarrow(M, \widetilde{g})$ is biharmonic non-harmonic. Moreover, in [19] the author gave some extensions of the result in [6] together with some further constructions of biharmonic maps. The author in [18] deform conformally the codomain metric in order to render a semi-conformal harmonic map biharmonic. In [3] the authors studied the case where $\phi:\left(M^{n}, g\right) \longrightarrow\left(N^{n}, h\right)$ is a conformal mapping between equidimensional manifolds where they show that a conformal mapping $\phi$ is biharmonic if and only if the gradient of its dilation satisfies a second order elliptic partial differential equation. We can refer the reader to [17], for a survey of biharmonic maps. In [16], the author obtain some results concerning harmonic maps and harmonic morphism on Kenmotsu manifolds, he prove that any structure preserving map from a Kenmotsu manifold to a Kahler manifold is harmonic and that there are no nonconstant harmonic holomorphic maps from a Kahler manifold to a Kenmotsu manifold. In this paper, we calculate the bitension field of $\psi:\left(N^{2 n}, J, h\right) \longrightarrow(M, \phi, \xi, \eta, g)$, where $\left(N^{2 n}, J, h\right)$ is a Kahlerian manifold and $(M, \phi, \xi, \eta, g)$ is a Kenmotsu manifold (Theorem 1), with this setting we obtain new example of biharmonic non-harmonic maps.

\section{Statement of results.}

As first result, we give another formula for the stress bi-energy tensor for a smooth map $\phi:\left(M^{m}, g\right) \rightarrow\left(N^{n}, h\right)$.

Proposition 1. Let $\phi:\left(M^{m}, g\right) \rightarrow\left(N^{n}, h\right)$ be a smooth map, then we have

$$
S_{2}(\phi)=\left(\frac{1}{2}|\tau(\phi)|^{2}+\operatorname{Tr}_{g} h(\nabla \tau(\phi), d \phi)\right) g-2 \operatorname{symh}(\nabla \tau(\phi), d \phi),
$$

and the trace of $S_{2}(\phi)$ is given by

$$
\operatorname{Tr}_{g} S_{2}(\phi)=\frac{m}{2}|\tau(\phi)|^{2}+(m-2) \operatorname{Tr}_{g} h(\nabla \tau(\phi), d \phi)
$$

Let $\left(N^{2 n}, J, h\right)$ be a Kahlerian manifold, $(M, \phi, \xi, \eta, g)$ be a Kenmotsu manifold and $\psi: N \longrightarrow M$ be a $(J, \phi)$-holomorphic map. To give a necessary and sufficient condition for the biharmonicity of the map $\psi$, we will calculate the bi-tension field $\tau_{2}(\psi)$. We get the following theorem : 
Theorem 1.Let $\left(N^{2 n}, J, h\right)$ be a Kahlerian manifold, $(M, \phi, \xi, \eta, g)$ be a Kenmotsu manifold and $\psi: N \longrightarrow M$ be a $(J, \phi)$ holomorphic map. Then, the bi-tension field of $\psi$ is given by

$$
\tau_{2}(\psi)=-2(\Delta(e(\psi)) \xi+2 d \psi(\operatorname{grad}(e(\psi))))
$$

The biharmonicity of $\psi$ is given by the following result :

Corollary 1. Let $\left(N^{2 n}, J, h\right)$ be a Kahlerian manifold, $(M, \phi, \xi, \eta, g)$ be a Kenmotsu manifold and $\psi: N \longrightarrow M$ be a $(\mathrm{J}, \phi)$-holomorphic map. Then $\psi$ is biharmonic if and only if

$$
\Delta(e(\psi)) \xi+2 d \psi(\operatorname{grad}(e(\psi)))=0 .
$$

By calculating of the term $\eta \circ \tau_{2}(\psi)$, we obtain immediately the following Corollary :

Corollary 2.Let $\left(N^{2 n}, J, h\right)$ be a Kahlerian manifold, $(M, \phi, \xi, \eta, g)$ be a Kenmotsu manifold and $\psi: N \longrightarrow M$ be a $(J, \phi)$ holomorphic map. If the map $\psi$ is biharmonic, then the function $e(\psi)$ is harmonic.

In particular, if the function $e(\psi)$ is constant we obtain the following result :

Corollary 3. Let $\left(N^{2 n}, J, h\right)$ be a Kahlerian manifold, $(M, \phi, \xi, \eta, g)$ be a Kenmotsu manifold and $\psi: N \longrightarrow M$ be a $(J, \phi)$-holomorphic map. If the function $e(\psi)$ is constant, then $\psi$ is biharmonic.

As an application of Theorem 1, we construct an example of biharmonic non-harmonic map.

Example 1. Let $\left(N=\mathbb{R}^{2}, J, h=d x^{2}+d y^{2}\right)$ be a complex manifold with complex structure $J\left(\frac{\partial}{\partial x}\right)=\frac{\partial}{\partial y}, J\left(\frac{\partial}{\partial y}\right)=-\frac{\partial}{\partial x}$ and $\left(M=\mathbb{R}^{3} \backslash\{(0,0,0)\}, \phi, \xi, \eta, g\right)$ be a Kenmotsu manifold with orthonormal basis

$$
\begin{gathered}
e_{1}=x_{3} \frac{\partial}{\partial x_{1}}, \\
e_{2}=x_{3} \frac{\partial}{\partial x_{2}}, \\
e_{3}=-x_{3} \frac{\partial}{\partial x_{3}} \\
\xi=e_{3} \\
\eta(X)=g(X, \xi),
\end{gathered}
$$

where

$$
g=\frac{1}{x_{3}^{2}}\left(d x_{1}^{2}+d x_{2}^{2}+d x_{3}^{2}\right)
$$

and

$$
\phi=\left(\begin{array}{ccc}
0 & 1 & 0 \\
-1 & 0 & 0 \\
0 & 0 & 0
\end{array}\right)
$$

Let $\psi: N \longrightarrow M$ be a $(J, \phi)$-holomorphic map defined by

$$
\psi(x, y)=\left(\psi_{1}(x, y), \psi_{2}(x, y), \psi_{3}(x, y)\right) .
$$

We have

$$
\frac{\partial \psi_{1}}{\partial x}=-\frac{\partial \psi_{2}}{\partial y}
$$




$$
\frac{\partial \psi_{1}}{\partial y}=\frac{\partial \psi_{2}}{\partial x}
$$

and

$$
\frac{\partial \psi_{3}}{\partial x}=\frac{\partial \psi_{3}}{\partial y}=0
$$

The last equation give $\psi_{3}(x, y)=C$, where $C$ is the real constant. A simple calculate give

$$
\begin{gathered}
e(\psi)=\frac{1}{C^{2}}\left(\left(\frac{\partial \psi_{1}}{\partial x}\right)^{2}+\left(\frac{\partial \psi_{1}}{\partial y}\right)^{2}\right), \\
\operatorname{grad}(e(\psi))=\frac{2}{C^{2}}\left(\frac{\partial \psi_{1}}{\partial x}\left(\frac{\partial^{2} \psi_{1}}{\partial x^{2}}+\frac{\partial^{2} \psi_{1}}{\partial x \partial y}\right) \frac{\partial}{\partial x}+\frac{\partial \psi_{1}}{\partial y}\left(\frac{\partial^{2} \psi_{1}}{\partial y^{2}}+\frac{\partial^{2} \psi_{1}}{\partial x \partial y}\right) \frac{\partial}{\partial y}\right), \\
d \psi(\operatorname{grad}(e(\psi)))=\frac{2}{C^{2}}\left(\left(\frac{\partial \psi_{1}}{\partial x}\right)^{2}\left(\frac{\partial^{2} \psi_{1}}{\partial x^{2}}+\frac{\partial^{2} \psi_{1}}{\partial x \partial y}\right)+\left(\frac{\partial \psi_{1}}{\partial y}\right)^{2}\left(\frac{\partial^{2} \psi_{1}}{\partial y^{2}}+\frac{\partial^{2} \psi_{1}}{\partial x \partial y}\right)\right) \frac{\partial}{\partial x_{1}} \\
+\frac{2}{C^{2}}\left(\frac{\partial \psi_{1}}{\partial x} \frac{\partial \psi_{2}}{\partial x}\left(\frac{\partial^{2} \psi_{1}}{\partial x^{2}}+\frac{\partial^{2} \psi_{1}}{\partial x \partial y}\right)+\frac{\partial \psi_{1}}{\partial y} \frac{\partial \psi_{2}}{\partial y}\left(\frac{\partial^{2} \psi_{1}}{\partial y^{2}}+\frac{\partial^{2} \psi_{1}}{\partial x \partial y}\right)\right) \frac{\partial}{\partial x_{2}}
\end{gathered}
$$

and

$$
\begin{aligned}
\Delta(e(\psi)) & =\frac{2}{C^{2}}\left(\left(\frac{\partial^{2} \psi_{1}}{\partial x^{2}}\right)^{2}+\left(\frac{\partial^{2} \psi_{2}}{\partial x^{2}}\right)^{2}+\left(\frac{\partial^{2} \psi_{1}}{\partial y^{2}}\right)^{2}+\left(\frac{\partial^{2} \psi_{2}}{\partial y^{2}}\right)^{2}\right) \\
& +\frac{2}{C^{2}}\left(\frac{\partial \psi_{1}}{\partial x}\left(\frac{\partial^{3} \psi_{1}}{\partial x^{3}}-\frac{\partial^{3} \psi_{2}}{\partial y^{3}}\right)+\frac{\partial \psi_{2}}{\partial x}\left(\frac{\partial^{3} \psi_{1}}{\partial y^{3}}+\frac{\partial^{3} \psi_{2}}{\partial x^{3}}\right)\right)
\end{aligned}
$$

In particular, if we consider $\psi_{1}(x, y)=\psi_{1}(x)$ and $\psi_{2}(x, y)=\psi_{2}(y)$, we obtain

$$
\psi(x, y)=(A x+B,-A y+D, C)
$$

where $A, B, C$ and $D$ are a real constants $(A, C \neq 0)$. In this case $e(\psi)=\frac{A^{2}}{C^{2}}$ and the map $\psi$ is biharmonic non-harmonic.

Proposition 2. Let $\left(N^{2 n}, J, h\right)$ be a Kahlerian manifold, $(M, \phi, \xi, \eta, g)$ be a Kenmotsu manifold and $\psi: N \longrightarrow M$ be a $(J, \phi)$-holomorphic map. Then, the stress bi-energy tensor of $\psi$ is given by

$$
S_{2}(\psi)=6(e(\psi))^{2} h-4 e(\psi) g(d \psi(\cdot), d \psi(\cdot)),
$$

where $g(d \psi(\cdot), d \psi(\cdot))(X, Y)=g(d \psi(X), d \psi(Y))$, and the trace of $S_{2}(\psi)$ is given by

$$
\operatorname{Tr}_{h} S_{2}(\psi)=(12 n-8)(e(\psi))^{2} .
$$

\section{Proof of results.}

Proof of Proposition 1. Let $\phi:\left(M^{m}, g\right) \rightarrow\left(N^{n}, h\right)$ be a smooth map. By definition, we have

$$
S_{2}(\phi)=\left(\frac{-1}{2}|\tau(\phi)|^{2}+\operatorname{divh}(\tau(\phi), d \phi)\right) g-2 \operatorname{symh}(\nabla \tau(\phi), d \phi) .
$$


Let $\left(e_{i}\right)_{i=1}^{m}$ is a local orthonormal frame field on $M$. For the term $\operatorname{divh}(\tau(\phi), d \phi)$, we have

$$
\begin{aligned}
\operatorname{divh}(\tau(\phi), d \phi) & =\left(\nabla_{e_{i}} h(\tau(\phi), d \phi)\right)\left(e_{i}\right) \\
& =e_{i}\left(h\left(\tau(\phi), d \phi\left(e_{i}\right)\right)\right)-h\left(\tau(\phi), d \phi\left(\nabla_{e_{i}} e_{i}\right)\right) \\
& =h\left(\nabla_{e_{i}}^{\phi} \tau(\phi), d \phi\left(e_{i}\right)\right)+h\left(\tau(\phi), \nabla_{e_{i}}^{\phi} d \phi\left(e_{i}\right)\right) \\
& -h\left(\tau(\phi), d \phi\left(\nabla_{e_{i}} e_{i}\right)\right) \\
& =h\left(\nabla_{e_{i}}^{\phi} \tau(\phi), d \phi\left(e_{i}\right)\right)+h\left(\tau(\phi), \nabla_{e_{i}}^{\phi} d \phi\left(e_{i}\right)-d \phi\left(\nabla_{e_{i}} e_{i}\right)\right) .
\end{aligned}
$$

But we know that

$$
h\left(\nabla_{e_{i}}^{\phi} \tau(\phi), d \phi\left(e_{i}\right)\right)=\operatorname{Tr}_{g} h(\nabla \tau(\phi), d \phi)
$$

and

$$
h\left(\tau(\phi), \nabla_{e_{i}}^{\phi} d \phi\left(e_{i}\right)-d \phi\left(\nabla_{e_{i}} e_{i}\right)\right)=h(\tau(\phi), \tau(\phi))=|\tau(\phi)|^{2}
$$

it follows that

$$
\operatorname{divh}(\tau(\phi), d \varphi)=\operatorname{Tr}_{g} h(\nabla \tau(\phi), d \phi)+|\tau(\phi)|^{2}
$$

If we replace (21) in (20), we obtain

$$
S_{2}(\phi)=\left(\frac{1}{2}|\tau(\phi)|^{2}+\operatorname{Tr}_{g} h(\nabla \tau(\phi), d \phi)\right) g-2 \operatorname{symh}(\nabla \tau(\phi), d \phi)
$$

By definition the trace of $S_{2}(\phi)$, we have

$$
\begin{aligned}
\operatorname{Tr}_{g} S_{2}(\phi) & =S_{2}(\phi)\left(e_{i}, e_{i}\right) \\
& =\left(\frac{1}{2}|\tau(\phi)|^{2}+\operatorname{Tr}_{g} h(\nabla \tau(\phi), d \phi)\right) g\left(e_{i}, e_{i}\right) \\
& -2 \operatorname{symh}(\nabla \tau(\phi), d \phi)\left(e_{i}, e_{i}\right) \\
& =m\left(\frac{1}{2}|\tau(\phi)|^{2}+\operatorname{Tr}_{g} h(\nabla \tau(\phi), d \phi)\right)-2 h\left(\nabla_{e_{i}} \tau(\phi), d \phi\left(e_{i}\right)\right) \\
& =\frac{m}{2}|\tau(\phi)|^{2}+m \operatorname{Tr}_{g} h(\nabla \tau(\phi), d \phi)-2 \operatorname{Tr}_{g} h(\nabla \tau(\phi), d \phi)
\end{aligned}
$$

which gives

$$
\operatorname{Tr}_{g} S_{2}(\phi)=\frac{m}{2}|\tau(\phi)|^{2}+(m-2) \operatorname{Tr}_{g} h(\nabla \tau(\phi), d \phi) .
$$

The proof of Proposition 1 is complete. To prove Theorem 1, we need the following Lemma :

Lemma 1. Let $\left(N^{2 n}, J, h\right)$ be a Kahlerian manifold, $(M, \phi, \xi, \eta, g)$ be a Kenmotsu manifold and $\psi: N \longrightarrow M$ be a $(J, \phi)$ holomorphic map. Then, we have for any $X \in \Gamma(T M)$

$$
(\eta \circ d \psi)(X)=0
$$

Proof of Lemma 1. Since $\psi: N \longrightarrow M$ is $(J, \phi)$-holomorphic map, then for any $X \in \Gamma(T N)$, we have

$$
(d \psi \circ J)(X)=(\phi \circ d \psi)(X)
$$


Using the fact that $\left(N^{2 n}, J, h\right)$ is a Kahlerian manifold, then

$$
J^{2}(X)=-X
$$

As $(M, \phi, \xi, \eta, g)$ is a Kenmotsu manifold and by using the equation 1, we have

$$
\eta \circ \phi=0
$$

Then, we obtain

$$
\begin{aligned}
(\eta \circ d \psi)(X) & =(\eta \circ d \psi)\left(-J^{2}(X)\right) \\
& =-\eta \circ d \psi \circ J(J(X)) \\
& =-\eta \circ \phi \circ d \psi(J(X)) \\
& =0 .
\end{aligned}
$$

We are now able to prove Theorem 1.

Proof of Theorem 1. By definition, we have

$$
\tau_{2}(\psi)=-\operatorname{Tr}_{h}\left(\nabla^{\psi}\right)^{2} \tau(\psi)-\operatorname{Tr}_{h} R^{M}(\tau(\psi), d \psi) d \psi
$$

The tension field of $\psi$ is given by (see [16])

$$
\tau(\psi)=2 e(\psi) \xi
$$

then

$$
\tau_{2}(\psi)=-2\left(\operatorname{Tr}_{h}\left(\nabla^{\psi}\right)^{2} e(\psi) \xi+\operatorname{Tr}_{h} R^{M}(e(\psi) \xi, d \psi) d \psi\right)
$$

In the first, we will simplify the term $\operatorname{Tr}_{h}\left(\nabla^{\psi}\right)^{2} e(\psi) \xi$. Let $\left(e_{i}\right)_{i=1}^{2 n}$ is a local orthonormal frame field on $N$, we have

$$
\operatorname{Tr}_{g}\left(\nabla^{\psi}\right)^{2} e(\psi) \xi=\nabla_{e_{i}}^{\psi} \nabla_{e_{i}}^{\psi} e(\psi) \xi-\nabla_{\nabla_{e_{i}}^{N} e_{i}}^{\psi} e(\psi) \xi
$$

A direct calculation gives

$$
\begin{aligned}
\nabla_{e_{i}}^{\psi} \nabla_{e_{i}}^{\psi} e(\psi) \xi & =\nabla_{e_{i}}^{\psi}\left(e(\psi) \nabla_{e_{i}}^{\psi} \xi\right)+\nabla_{e_{i}}^{\psi}\left(e_{i}(e(\psi)) \xi\right) \\
& =e(\psi) \nabla_{e_{i}}^{\psi} \nabla_{e_{i}}^{\psi} \xi+e_{i}(e(\psi)) \nabla_{e_{i}}^{\psi} \xi+e_{i}(e(\psi)) \nabla_{e_{i}}^{\psi} \xi \\
& +e_{i}\left(e_{i}(e(\psi))\right) \xi \\
& =e(\psi) \nabla_{e_{i}}^{\psi} \nabla_{e_{i}}^{\psi} \xi+2 \nabla_{\operatorname{grad}(e(\psi))}^{\psi} \xi+e_{i}\left(e_{i}(e(\psi))\right) \xi
\end{aligned}
$$

and

$$
\nabla_{\nabla_{e_{i}} e_{i}}^{\psi} e(\psi) \xi=e(\psi) \nabla_{\nabla_{e_{i}} e_{i}}^{\psi} \xi+\nabla_{e_{i}} e_{i}(e(\psi)) \xi
$$

Using the fact that

$$
\Delta(e(\psi))=e_{i}\left(e_{i}(e(\psi))\right)-\left(\nabla_{e_{i}}^{N} e_{i}\right)(e(\psi))
$$

and

$$
\operatorname{Tr}_{g}\left(\nabla^{\psi}\right)^{2} \xi=\nabla_{e_{i}}^{\psi} \nabla_{e_{i}}^{\psi} \xi-\nabla_{\nabla_{e_{i}}^{N} e_{i}}^{\psi} \xi
$$


we deduce that

$$
\begin{aligned}
\operatorname{Tr}_{h}\left(\nabla^{\psi}\right)^{2} e(\psi) \xi & =e(\psi) \operatorname{Tr}_{h}\left(\nabla^{\psi}\right)^{2} \xi+\Delta(e(\psi)) \xi \\
& +2 \nabla_{\operatorname{grad}(e(\psi))}^{\psi} \xi
\end{aligned}
$$

Calculate the term $\operatorname{Tr}_{h}\left(\nabla^{\psi}\right)^{2} \xi$, we have

$$
\operatorname{Tr}_{h}\left(\nabla^{\psi}\right)^{2} \xi=\nabla_{e_{i}}^{\psi} \nabla_{e_{i}}^{\psi} \xi-\nabla_{\nabla_{e_{i}} e_{i}}^{\psi} \xi
$$

By using equation (6), we obtain

$$
\nabla_{e_{i}}^{\psi} \xi=\nabla_{d \psi\left(e_{i}\right)} \xi=d \psi\left(e_{i}\right)-(\eta \circ d \psi)\left(e_{i}\right) \xi
$$

and by Lemma 1, we have

$$
(\eta \circ d \psi)\left(e_{i}\right)=0
$$

then

$$
\nabla_{e_{i}}^{\psi} \xi=d \psi\left(e_{i}\right)
$$

wish gives us

$$
\nabla_{e_{i}}^{\psi} \nabla_{e_{i}}^{\psi} \xi=\nabla_{e_{i}}^{\psi} d \psi\left(e_{i}\right)
$$

and

$$
\begin{aligned}
\nabla_{\nabla_{e_{i}} e_{i}}^{\psi} \xi & =\nabla_{d \psi\left(\nabla_{e_{i}} e_{i}\right)} \xi \\
& =d \psi\left(\nabla_{e_{i}} e_{i}\right)-(\eta \circ d \psi)\left(\nabla_{e_{i}} e_{i}\right) \xi \\
& =d \psi\left(\nabla_{e_{i}} e_{i}\right),
\end{aligned}
$$

we conclude that

$$
\operatorname{Tr}_{h}\left(\nabla^{\psi}\right)^{2} \xi=\nabla_{e_{i}}^{\psi} d \psi\left(e_{i}\right)-d \psi\left(\nabla_{e_{i}} e_{i}\right)=\tau(\psi)
$$

Then

$$
\operatorname{Tr}_{h}\left(\nabla^{\psi}\right)^{2} \xi=2 e(\psi) \xi
$$

Finally, we obtain

$$
\operatorname{Tr}_{h}\left(\nabla^{\psi}\right)^{2} e(\psi) \xi=2(e(\psi))^{2} \xi+\Delta(e(\psi)) \xi+2 \nabla_{\operatorname{grad}(e(\psi))}^{\psi} \xi
$$

Now simplify the term $\nabla_{\operatorname{grad}(e(\psi))}^{\psi} \xi$, we have

$$
\begin{aligned}
\nabla_{\operatorname{grad}(e(\psi))}^{\psi} \xi & =\nabla_{d \psi(\operatorname{grad}(e(\psi)))}^{M} \\
& =d \psi(\operatorname{grad}(e(\psi)))-\eta(d \psi(\operatorname{grad}(e(\psi)))) \xi \\
& =d \psi(\operatorname{grad}(e(\psi)))-(\eta \circ d \psi)(\operatorname{grad}(e(\psi))) \xi \\
& =d \psi(\operatorname{grad}(e(\psi))) .
\end{aligned}
$$

which finally gives us

$$
\operatorname{Tr}_{h}\left(\nabla^{\psi}\right)^{2} e(\psi) \xi=2(e(\psi))^{2} \xi+\Delta(e(\psi)) \xi+2 d \psi(\operatorname{grad}(e(\psi)))
$$

To complete the proof, look at the term $\operatorname{Tr}_{g} R^{M}(e(\psi) \xi, d \psi) d \psi$, we have 


$$
\begin{aligned}
\operatorname{Tr}_{h} R^{M}(e(\psi) \xi, d \psi) d \psi & =e(\psi) \operatorname{Tr}_{h} R^{M}(\xi, d \psi) d \psi \\
& =e(\psi) R^{N}\left(\xi, d \psi\left(e_{i}\right)\right) d \psi\left(e_{i}\right) .
\end{aligned}
$$

Using equation (9), we obtain

$$
\begin{aligned}
R^{N}\left(\xi, d \psi\left(e_{i}\right)\right) d \psi\left(e_{i}\right) & =\eta\left(d \psi\left(e_{i}\right)\right)-g\left(d \psi\left(e_{i}\right), d \psi\left(e_{i}\right)\right) \xi \\
& =(\eta \circ d \psi)\left(e_{i}\right)-2 e(\psi) \xi \\
& =-2 e(\psi) \xi
\end{aligned}
$$

Then

$$
\operatorname{Tr}_{h} R^{N}(e(\psi) \xi, d \psi) d \psi=-2(e(\psi))^{2} \xi
$$

If we replace (25) and (26) in (22), wa arrive at

$$
\tau_{2}(\psi)=-2(\Delta(e(\psi)) \xi+2 d \psi(\operatorname{grad}(e(\psi))))
$$

This completes the proof of Theorem 1 . Then $\psi: N \longrightarrow M$ is biharmonic if and only if

$$
\Delta(e(\psi)) \xi+2 d \psi(\operatorname{grad}(e(\psi)))=0
$$

Proof of Corollary 2. If we assume that the map $\psi: N \longrightarrow M$ is biharmonic, by Theorem 1, we have

$$
\Delta(e(\psi)) \xi+2 d \psi(\operatorname{grad}(e(\psi)))=0
$$

Then

$$
\eta \circ(\Delta(e(\psi)) \xi+2 d \psi(\operatorname{grad}(e(\psi))))=0,
$$

it follows that

$$
\Delta(e(\psi)) \eta(\xi)+2(\eta \circ d \psi)(\operatorname{grad}(e(\psi)))=0 .
$$

By using the equation (1), we have

$$
\eta(\xi)=1
$$

and by Lemma 1, we have

$$
(\eta \circ d \psi)(\operatorname{grad}(e(\psi)))=0
$$

Which finally gives

$$
\Delta(e(\psi))=0 .
$$

We deduce that the function $e(\psi)$ is harmonic.

Proof of Proposition 2. By definition, for any $X, Y \in \Gamma(T N)$ we have

$$
\begin{aligned}
S_{2}(\psi)(X, Y) & =\left(\frac{1}{2}|\tau(\psi)|^{2}+\operatorname{Tr}_{h} g(\nabla \tau(\psi), d \psi)\right) h(X, Y) \\
& -2 \operatorname{symg}(\nabla \tau(\psi), d \psi)(X, Y) .
\end{aligned}
$$


Since

$$
\tau(\psi)=2 e(\psi) \xi
$$

it follows that

$$
|\tau(\psi)|^{2}=4(e(\psi))^{2}
$$

For the term $\operatorname{Tr}_{h} g(\nabla \tau(\psi), d \psi)$, we have

$$
\begin{aligned}
\operatorname{Tr}_{h} g(\nabla \tau(\psi), d \psi) & =2 g\left(\nabla_{e_{i}} e(\psi) \xi, d \psi\left(e_{i}\right)\right) \\
& =2 e(\psi) g\left(\nabla_{e_{i}}^{\psi} \xi, d \psi\left(e_{i}\right)\right) \\
& +2 e_{i}(e(\psi)) g\left(\xi, d \psi\left(e_{i}\right)\right)
\end{aligned}
$$

where $\left(e_{i}\right)_{i=1}^{2 n}$ is a local orthonormal frame field on $N$. By equation (6), we obtain

$$
\nabla_{e_{i}}^{\psi} \xi=\nabla_{d \psi\left(e_{i}\right)}^{M} \xi=d \psi\left(e_{i}\right)-\eta\left(d \psi\left(e_{i}\right)\right) \xi=d \psi\left(e_{i}\right)
$$

and by (3), we have

$$
g\left(\xi, d \psi\left(e_{i}\right)\right)=\eta\left(d \psi\left(e_{i}\right)\right)=0
$$

then

$$
\operatorname{Tr}_{h} g(\nabla \tau(\psi), d \psi)=2 e(\psi) g\left(d \psi\left(e_{i}\right), d \psi\left(e_{i}\right)\right)=4(e(\psi))^{2}
$$

it follow that

$$
\frac{1}{2}|\tau(\psi)|^{2}+\operatorname{Tr}_{h} g(\nabla \tau(\psi), d \psi)=6(e(\psi))^{2}
$$

Finally, for the term $\operatorname{symg}(\nabla \tau(\psi), d \psi)(X, Y)$, we have

$$
\operatorname{symg}(\nabla \tau(\psi), d \psi)(X, Y)=\frac{1}{2} g\left(\nabla_{X}^{\psi} \tau(\psi), d \psi(Y)\right)+\frac{1}{2} g\left(\nabla_{Y}^{\psi} \tau(\psi), d \psi(X)\right) .
$$

A simple calculate gives

$$
\begin{aligned}
\frac{1}{2} g\left(\nabla_{X}^{\psi} \tau(\psi), d \psi(Y)\right) & =g\left(\nabla_{X}^{\psi} e(\psi) \xi, d \psi(Y)\right) \\
& =e(\psi) g\left(\nabla_{X}^{\psi} \xi, d \psi(Y)\right)+X(e(\psi)) g(\xi, d \psi(Y))
\end{aligned}
$$

but by (6), we have

$$
\nabla_{X}^{\psi} \xi=\nabla_{d \psi(X)}^{M} \xi=d \psi(X)-\eta(d \psi(X)) \xi=d \psi(X)
$$

and by (3), we obtain

$$
g(\xi, d \psi(Y))=\eta(d \psi(Y))=0,
$$

then

$$
\frac{1}{2} g\left(\nabla_{X}^{\psi} \tau(\psi), d \psi(Y)\right)=g(d \psi(X), d \psi(Y))
$$

A similar calculate gives

$$
\frac{1}{2} g\left(\nabla_{Y}^{\psi} \tau(\psi), d \psi(X)\right)=g(d \psi(X), d \psi(Y))
$$

We deduce that

$$
\operatorname{symg}(\nabla \tau(\psi), d \psi)(X, Y)=2 g(d \psi(X), d \psi(Y)),
$$


then

$$
S_{2}(\psi)(X, Y)=6(e(\psi))^{2} g(X, Y)-4 g(d \psi(X), d \psi(Y)) .
$$

finally, the stress bi-energy tensor is given by

$$
S_{2}(\psi)=6(e(\psi))^{2} g-4 g(d \psi(\cdot), d \psi(\cdot))
$$

where $g(d \psi(\cdot), d \psi(\cdot))(X, Y)=g(d \psi(X), d \psi(Y))$. Now calculate $\operatorname{Tr}_{h} S_{2}(\psi)$, we have

$$
\begin{aligned}
\operatorname{Tr}_{h} S_{2}(\psi) & =S_{2}(\psi)\left(e_{i}, e_{i}\right) \\
& =6(e(\psi))^{2} g\left(e_{i}, e_{i}\right)-4 g\left(d \psi\left(e_{i}\right), d \psi\left(e_{i}\right)\right) \\
& =12 n(e(\psi))^{2}-4|d \psi|^{2} \\
& =12 n(e(\psi))^{2}-8(e(\psi))^{2} \\
& =(12 n-8)(e(\psi))^{2} .
\end{aligned}
$$

We conclude that $\operatorname{Tr}_{g} S_{2}(\psi)=0$ if and only if the map $\psi$ is constant.

\section{References}

[1] P. Baird, Harmonic maps with symmetry, harmonic morphisms and deformation of metrics, Pitman Books Limited, 27-39, (1983).

[2] P. Baird and J. Eells, A conservation law for harmonic maps, Lecture Notes in Math. 894, Springer, 1-25, (1981).

[3] P.Baird, A. Fardoun and S. Ouakkas, Conformal and semi-conformal biharmonic maps, Ann. Glob Anal Geom 34, 403-414 (2008).

[4] P. Baird and D. Kamissoko, On constructing biharmonic maps and metrics, Annals of Global Analysis and Geometry 23, 65-75, (2003).

[5] P. Baird and J.C. Wood, Harmonic morphisms between Riemannain manifolds, Oxford Sciences Publications (2003).

[6] A. Balmus, Biharmonic properties and conformal changes, An. Stiint. Univ. Al.I. Cuza Iasi Mat. (N.S.) 50, 367-372, (2004).

[7] D.E. Blair, Riemannian geometry of contact and Symplectic Manifolds, Birkhauser. Boston, Second Edition (2010).

[8] U.C.De and G. Pathok, On 3-dimensional Kenmotsu manifolds, Indian J. Pure Appl. Math. 35, 159-165, (2004).

[9] J. Eells and L. Lemaire, A report on harmonic maps, Bull. London Math. Soc. 16, 1-68, (1978).

[10] J. Eells and L. Lemaire, Another report on harmonic maps, Bull. London Math. Soc. 20, 385-524, (1988).

[11] J. Eells and L. Lemaire, Selected topics in harmonic maps, CNMS Regional Conference Series of the National Sciences Foundation, November 1981.

[12] J. Eells and A. Ratto, Harmonic Maps and Minimal Immersions with Symmetries, Princeton University Press 1993.

[13] G. Y. Jiang, 2-harmonic maps and their first and second variational formulas, Chinese Ann. Math. Ser. A 7, 389-402, (1986).

[14] J.B Jun, U.C. De and G. Pathak, On Kenmotsu Manifolds, J. Korean Math. Soc. 42, No. 3, 435-445, (2005).

[15] K. Kenmotsu, A class of almost contact Riemannian manifolds, Tohoku Math. J. II Ser. 24, 93-103, (1972).

[16] A. Najma, Harmonic Maps on Kenmotsu Manifolds, An. St. Univ. Ovidius Constanta. Vol. 21(3), 197-208, 2013.

[17] C. Oniciuc, New examples of biharmonic maps in spheres, Colloq. Math., 97, 131-139, (2003).

[18] Ouakkas, S, Biharmonic maps, conformal deformations and the Hopf maps, Diff. Geom. Appl, 26, 495-502, (2008).

[19] Y.-L. Ou, p-harmonic morphisms, biharmonic morphisms, and non-harmonic biharmonic maps, J. Geom. Phys. Volume 56, 3 , 358-374, (2006).

[20] G. Pitis, Geometry of Kenmotsu manifolds, Publishing House of Transilvania University of Brassov, Bra sov, (2007).

[21] K. Yano and M. Kon, Structures on manifolds, vol. 3, Series in pure Math., World Scientifc, Singapore, 1984. 